\title{
Anomalous origin of the left coronary artery from the pulmonary artery: natural history and normal pregnancies
}

\author{
A K Nightingale, C J Burrell, A J Marshall
}

\begin{abstract}
Two female patients are described with anomalous origin of the left coronary artery arising from the pulmonary artery who sustained an anterolateral myocardial infarction in infancy. Neither patient received surgical treatment although both have lived to middle age with minimal cardiovascular problems and have had uncomplicated pregnancies. Good exercise tolerance and long term survival may be possible even without surgery for patients with this anomaly.

(Heart 1998;80:629-631)
\end{abstract}

Keywords: myocardial infarction; coronary vessel anomalies; pulmonary artery; pregnancy; congenital heart disease

Anomalous origin of the left coronary artery arising from the pulmonary artery is a rare congenital abnormality ${ }^{1}$ affecting one in 300000 live births. It may cause myocardial infarction within a few weeks of birth and can result in impaired left ventricular function, mitral regurgitation, and progressive heart failure. Optimal management has been debated with a bias to early surgery to restore a two coronary artery system in the hope of preventing sudden death and restoring left ventricular function. ${ }^{2}$

We describe two female patients with this anomaly who sustained an anterolateral myocardial infarction in infancy. Neither received surgical treatment but both have lived to middle age and have had uncomplicated pregnancies. We discuss their outcome and possible treatment strategies.

South West

Cardiothoracic

Centre, Plymouth

Hospitals NHS Trust,

Derriford Hospital,

Plymouth PL6 8DH,

UK

A K Nightingale

C J Burrell

A J Marshall

Correspondence to:

Dr Nightingale.

Accepted for publication 25 March 1998
On review aged 12 months an anomalous left coronary artery was suspected but her parents declined further investigation. She remained well.
At age 10 years her heart was not clinically enlarged but she had a short ejection systolic murmur at the left sternal edge, a third heart sound, and reversed splitting of the second heart sound. Electrocardiography showed an old anterolateral myocardial infarction.

At age 19 multigated nuclear angiography showed an ejection fraction of $45 \%$ with poor wall motion at the apex. She was referred to the National Heart Hospital in London, UK where cardiac catheterisation showed a normal right coronary with a good left ventricle but no left coronary artery arising from the aorta (fig 1). The left coronary artery was seen to fill retrogradely from the right coronary injection (fig 1) draining into the pulmonary artery. There was a small left to right shunt (1.25:1). The septum and part of the anterior wall of the left ventricle were dyskinetic but not frankly aneurysmal. She was advised surgery but declined.

Aged 23 she became pregnant. She had an emergency caesarian section at term because of failure to progress. She became bradycardic on induction of anaesthesia with etomidate and suxamethonium, and electrocardiography showed sinus node arrest followed by an idioventricular escape rhythm with a rate of 30 beats $/ \mathrm{min}$. She was given $0.4 \mathrm{mg}$ of atropine and her heart rate increased to 75 beats/min. After 40 seconds she reverted to sinus rhythm at a rate of 100 beats $/ \mathrm{min}$.

She had one further pregnancy, which was uncomplicated but was delivered by elective caesarian section. She was subsequently sterilised and remains well on no medication.

\section{Case 2}

This patients is now 42 years old. Following recurrent bronchitis in infancy cardiomegaly was noted on chest radiography. Aged 3 years she was investigated at the Brompton Hospital, London, UK where electrocardiography showed evidence of an anterolateral myocardial infarction, and cardiac catheterisation showed an aneurysm at the left ventricular apex with the probable diagnosis of anomalous origin of the left coronary artery from the pulmonary artery.

A repeat cardiac catheterisation aged 12 showed left ventricular end diastolic pressure 

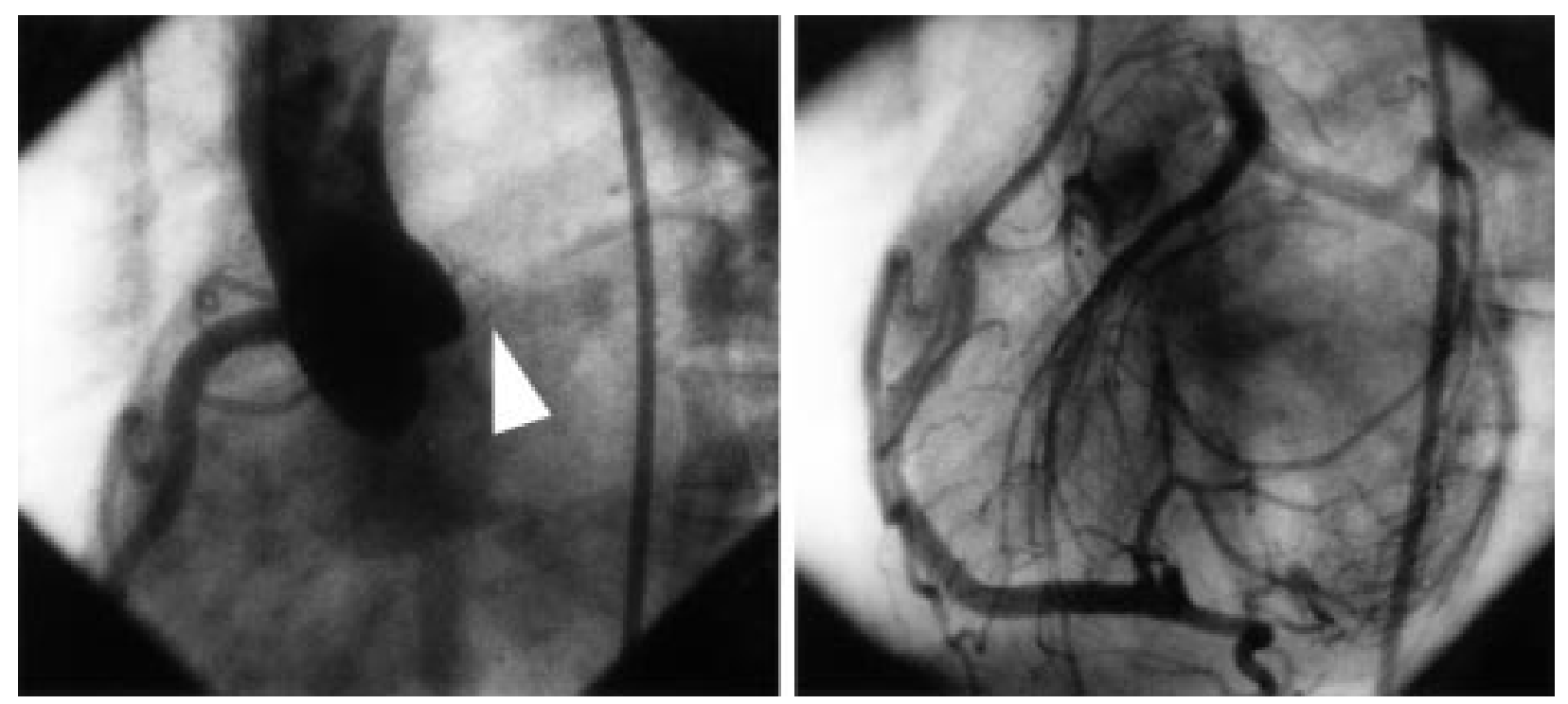

Figure 1 (Left) Aortogram of case 1 showing a normal right coronary artery arising from the aorta but absence of the left coronary artery (arrowed). (Right) Selective injection into the right coronary artery shows retrograde filling of the left anterior descending and circumflex arteries through collaterals with the right coronary artery. The left system then drains into the pulmonary artery.

$10-25 \mathrm{~mm} \mathrm{Hg}$, pulmonary artery pressure $45 / 20 \mathrm{~mm} \mathrm{Hg}$ (mean $22 \mathrm{~mm} \mathrm{Hg}$ ), and right ventricular end diastolic pressure 8-17 mm Hg. Left ventricular angiography showed an apical aneurysm and filled a large tortuous right coronary artery arising from the aorta while the left coronary artery filled draining into the pulmonary artery. She was not treated. She had two pregnancies with normal deliveries and no cardiovascular problems.

Reinvestigated aged 34, she was found to have a large left to right shunt with a pulmonary artery saturation of $93 \%$ but normal right heart pressures. Right coronary angiography showed a huge artery, which supplied the territory of the left coronary artery and drained into the pulmonary artery. Left ventricular angiography showed a large area of antero-apical akinesia.

Surgery was recommended on the basis of the large left to right shunt causing volume overload on a damaged ventricle but she declined.

She remains asymptomatic and on no medication. Clinically she is in sinus rhythm with a soft apical systolic murmur but no third or fourth heart sounds and no pulmonary oedema. Chest radiography shows slight enlargement of the cardiac silhouette and electrocardiography shows left axis deviation and anteroseptal Q waves. Echocardiography demonstrates persistent akinesia of the apex of the left ventricle and mild mitral regurgitation.

\section{Discussion}

Anomalous origin of the left coronary artery from the pulmonary artery usually has serious consequences early in life. Mortality varies in different series. In two series of surgically treated patients diagnosed younger than 6 months, nine of $21^{2}$ and eight of 23 died. ${ }^{3}$ Arciniegas et al reported a mortality of eight of 25 patients but most deaths were in a non-surgically treated group (six of six) with a surgical mortality of two of $19 .{ }^{4}$ Wesselhoeft et al reviewed the literature of infants with a diagnosis of anomalous origin of the left coronary artery from the pulmonary artery who had symptoms of angina or cardiomyopathy and found a mortality of 109 of $116^{5}$; however, many of these patients were diagnosed postmortem. A history was available in only 60 patients so no analysis of surgical versus non-surgical treatment was attempted.

In an uncommon condition that is difficult to diagnose in life, retrospective studies will show an excess of deaths in an "untreated" group as the diagnosis is not made in those who survive. The other difficulty is that the small numbers involved mean that the power to detect a real difference in treatment groups is extremely low. Furthermore, prospective randomised studies are virtually impossible again because of the small numbers.

Some authors have recently made a strong case for surgery. Kakou Guikahaue et al recommend early operation while Driscoll et al ${ }^{\beta}$ suggest delaying until at least 18 months of age and if possible until 5 to 7 years of age. If surgery is to be recommended, which procedure should be used? Initial work was done with simple ligation of the anomalous coronary artery at its origin from the pulmonary artery. ${ }^{6}$ This seemed to work well in some cases and was a fairly straightforward operation. However, if the flow was from right to left, ligation of the artery caused catastrophic consequences and very high mortality. Later work has been done using a saphenous vein graft from the aorta to the pulmonary origin of the anomalous artery, which is then ligated from the pulmonary artery restoring the flow of oxygenated blood. Other techniques have been developed, including retroaortic aortocoronary bypass with a free segment of the left subclavian artery and transpulmonary aortocoronary bypass with the left subclavian artery. ${ }^{4}$

There are theoretical advantages to surgery. These include re-establishing a two coronary artery system and restoring oxygenated blood 
flow to the left ventricular myocardium. Somerville and Ross have demonstrated well the physiological benefits of surgery in a single case. $^{7}$

Most authors agree that initial medical treatment should be as for an acute myocardial infarction. Can we extrapolate from our knowledge of patients with acute myocardial infarction? Both of our patients sustained an anterior myocardial infarction with some left ventricular impairment. They are both free from symptoms. If we consider their anomalous left coronary artery analogous to a proximal circumflex and proximal left anterior descending artery lesion with an ejection fraction of over $50 \%$, then their 15 year survival will be $71 \%$ with surgery and $67 \%$ without. ${ }^{8}$ It is known that younger patients with myocardial infarction have a better prognosis and show a different pattern of coronary disease from an older group. ${ }^{9}$

What advice can we give our adult patients with non-surgically treated anomalous left coronary artery arising from the pulmonary artery? Many will be young women wishing to become pregnant. Our two cases suggest that pregnancy is certainly possible. Care must be taken during labour, especially if anaesthesia is induced with drugs that may reduce cardiac output. There is a risk of sudden cardiac death in these patients and they may benefit from an implantable cardiac defibrillator. Angiotensin converting enzyme inhibitors may have a role in modification of ventricular remodelling but should be avoided in pregnancy.

We present long term follow up data for two females over a period of 41 and 30 years, respectively. They both have tolerated well the haemodynamic stress of several pregnancies although one of four pregnancies was complicated by transient sinus node arrest on induction of anaesthesia. These two cases show that good exercise tolerance and long term survival may be possible even without surgery for patients with anomalous left coronary artery arising from the pulmonary artery.

We thank Dr Jane Somerville, Royal Brompton Hospital, for her help in managing these complicated patients and for her advice in preparing this report.

1 Bland EF, White PD, Garland J. Congenital anomalies of coronary arteries; report of an unusual case associated with cardiac hypertrophy. Am f Cardiol 1933;8:787-801.

2 Kakou Guikahue M, Sidi D, Kachaner J, et al. Anomalous left coronary artery arising from the pulmonary artery in infancy: is early operation better? Br Heart $\mathcal{F} 1988$; 60:5226.

3 Driscoll DJ, Nihill MR, Mullins CE, et al. Management of symptomatic infants with anomalous origin of the left coronary artery from the pulmonary artery. Am 7 Cardiol onary artery from

4 Arciniegas E, Farooki Z, Hakimi M, et al. Management of anomalous left coronary artery from the pulmonary artery. Circulation 1980;62(suppl I): 180-9.

5 Wesselhoeft H, Fawcett JS, Johnson AL. Anomalous origin of the left coronary artery from the pulmonary trunk. Its clinical spectrum, pathology and pathophysiology, based on review of 140 cases with seven further cases. Circulation

6 Wilson CL, Dlabal PW, McGuire MD. Surgical treatment of anomalous left coronary artery from pulmonary artery: follow-up in teenagers and adults. Am Heart $\mathcal{f}$ 1979;98: $440-5$.

7 Somerville J, Ross DN. Left coronary artery from the pulmonary artery. Physiological considerations of surgical correction. Thorax 1970;25:207-12.

8 Caracciolo EA, Davis KB, Sopko G, et al. Comparison of surgical and medical group survival in patients with left main equivalent coronary artery disease. Long-term CASS main equivalent coronary artery disease.

9 Zimmerman FH, Cameron A, Fisher LD, et al. Myocardial infarction in young adults: angiographic characterisation, risk factors and prognosis (coronary artery surgery study registry). F Am Coll Cardiol 1995;26:654-61. 